\title{
Viremia, CTCAE
}

National Cancer Institute

\section{Source}

National Cancer Institute. Viremia, CT CAE. NCI Thesaurus. Code C143215.

A disorder characterized by the presence of a virus in the blood stream. 